International Journal of Pure and Applied Mathematics

Volume 99 No. 2 2015, 201-204

ISSN: 1311-8080 (printed version); ISSN: 1314-3395 (on-line version)

url: http://www.ijpam.eu

doi: http://dx.doi.org/10.12732/ijpam.v99i2.7

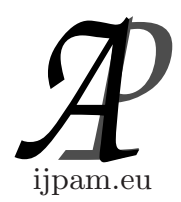

\title{
CYCLICITY OF WEIGHTED COMPOSITION OPERATORS ON SOME BK SPACE
}

\author{
B. Yousefi ${ }^{1}$, S.M.A. Musavi ${ }^{2}$ \\ ${ }^{1,2}$ Department of Mathematics \\ Payame Noor University \\ P.O. Box 19395-3697, Tehran, IRAN
}

\begin{abstract}
We will investigate the cyclicity for the adjoint of a weighted composition operator acting on $\left(\hat{l_{p}}(\alpha)\right)^{*}$.
\end{abstract}

AMS Subject Classification: 47B37, 46A25

Key Words: AK space, BK space, weighted composition operator, multipliers, cyclic vector, bounded point evaluation

\section{Introduction}

We write $\omega$ for the set of all complex sequences $x=\left(x_{k}\right)_{k=0}^{\infty}$. Let $\phi, l_{\infty}$ and $c_{0}$ denote the set of all finite, bounded and null sequences. We write

$$
l_{p}=\left\{x \in \omega: \sum_{k=0}^{\infty}\left|x_{k}\right|^{p}<\infty\right\}
$$

for $1 \leq p<\infty$. By $e^{(n)}\left(n \in N_{0}\right)$, we denote the sequence with $e_{n}^{(n)}=1$ and $e_{k}^{(n)}=0$ whenever $k \neq n$. For any sequence $x=\left(x_{k}\right)_{k=0}^{\infty}$, let $x^{[n]}=\sum_{k=0}^{n} x_{k} e^{(k)}$ be its n-section. Given any subset $F$ of $\omega$, we write $\hat{F}$ for the set of all formal power series $\hat{f}$ with $\hat{f}(z)=\sum_{k=0}^{\infty} f_{k} z^{k}$ where $f=\left(f_{k}\right)_{k=0}^{\infty} \in F$, regardless of whether or not the series converges for any value of z. Let $\hat{M}_{z}: \hat{F} \rightarrow \hat{\omega}$ be defined by $\left(\hat{M}_{z} \hat{f}\right)=\sum_{k=0}^{\infty} f_{k} z^{k+1}$.

Received: November 13, 2014

(C) 2015 Academic Publications, Ltd. url: www.acadpubl.eu

${ }^{\S}$ Correspondence author 
A $B K$ space is a Banach sequence space with the property that convergence implies coordinatewise convergence. A $B K$ space $F$ containing $\phi$ is said to have $A K$ if every sequence $f=\left(f_{k}\right)_{k=0}^{\infty} \in F$ has a unique representation $f=$ $\sum_{k=0}^{\infty} f_{k} e^{(k)}$, that is $f=\lim _{n \rightarrow \infty} f^{[n]} ;$ it is said to have $A D$, if $\phi$ is dense in $F$. Given any subset $F$ of $\omega$, the set

$$
F^{\beta}=\left\{a \in \omega: \sum_{k=0}^{\infty} a_{k} f_{k} \quad \text { converges for all } f \in F\right\}
$$

is called the $\beta$-dual of $F$.

Let $F$ be a normed sequence space and $\hat{F}$ be the space of formal power series with coefficients in $F$ endowed with the norm of $F$. Then $F$ and $\hat{F}$ are norm isomorphic.

We say that a vector $x$ in a Banach space $X$ is a cyclic vector of a bounded operator $A$ on $X$ if $X=\operatorname{span}\left\{A^{n} x: n=0,1,2, \ldots\right\}$.

Consider $f=\left\{f_{k}\right\}_{k=0}^{\infty}$ and $g=\left\{g_{k}\right\}_{k=0}^{\infty}$ in $\omega$ and let $E \subset \omega$. Define $f g=\left\{f_{k} g_{k}\right\}_{k=0}^{\infty}$ and

$$
g^{-1} \star E=\{f \in \omega: f g \in E\} .
$$

If $\alpha=\left\{\alpha_{k}\right\}_{k=0}^{\infty} \in \omega$ is a given sequence with $\alpha_{k} \neq 0$ for all $k$, then by $1 / \alpha$ we mean $1 / \alpha=\left\{1 / \alpha_{k}\right\}_{k=0}^{\infty}$. Write $\hat{F}(\alpha)=\left(\alpha^{-1} \star F\right)$ for any subset $F$ of $\omega$. From now on we suppose that $\alpha=\left\{\alpha_{k}\right\}_{k=0}^{\infty} \in \omega$ satisfying $\alpha_{0}=1$ and $\alpha_{k} \neq 0$ for all $k \geq 1$. Note that the space $\hat{l}_{p}(\alpha)$ is a reflexive Banach space and the dual of $\hat{l}_{p}(\alpha)$ is $\hat{l}_{q}\left(\alpha^{-1}\right)$.

If $\lambda$ is a complex number, then $e(\lambda)$ denotes the functional of evaluation at $\lambda$, defined on $\hat{l}_{p}(\alpha)$ by $e(\lambda)(\hat{f})=\hat{f}(\lambda)$.

A complex valued function $\varphi$ on $\Omega$ for which $\varphi \hat{f} \in \hat{F}$ for every $\hat{f} \in \hat{F}$ is called a multiplier of $\hat{F}$ and the collection of all these multipliers is denoted by $\mathcal{M}(\hat{F})$. For some sources on sequence spaces, see $[1-6]$.

\section{Main Results}

In this section we will investigate the cyclicity of the adjoint of weighted composition operators acting on $\hat{l}_{p}(\alpha)^{*}$. By $U$ we mean the open unit disc.

Lemma 1. A complex number $\lambda$ is a bounded point evaluation on $\hat{l}_{p}(\alpha)$ if and only if $\left\{\lambda^{n}\right\}_{n=0}^{\infty} \in l_{q}\left(\alpha^{-1}\right)$.

Proof. Note that $\lambda$ is a bounded point evaluation on $\hat{l}_{p}(\alpha)$ if and only if the functional $e(\lambda)$ is bounded on $\hat{l}_{p}(\alpha)$. But the dual of $\hat{l}_{p}(\alpha)$ is $\hat{l}_{q}\left(\alpha^{-1}\right)$ and we 
can see that $e(\lambda)\left(\left(e^{(k)} \hat{)}\right)=\left(e^{(k)} \hat{)}(\lambda)=\lambda^{k}\right.\right.$ for all integers $k \geq 0$. This completes the proof.

Theorem 2. Let each point of $U$ is a bounded point evaluation on $\hat{l}_{p}(\alpha)$. Then a polynomial $\hat{p}$ is cyclic for $\hat{M}_{z}$ if and only if $\hat{p}$ vanishes at no point in $U$.

Proof. Let $\hat{p}(z)=\left(z-\lambda_{1}\right) \ldots\left(z-\lambda_{m}\right)$ be such that $\lambda_{i} \notin U$ for $i=1, \ldots, m$. Fix $k \in\{1, \ldots, m\}$ and consider $M_{k} \in \hat{l}_{q}\left(\alpha^{-1}\right)$ satisfying $\left.M_{k}\left(\hat{M}_{z}\right)^{n}\left(z-\lambda_{k}\right)\right)=0$ for all integers $n \geq 0$. So there exists $h \in l_{q}\left(\alpha^{-1}\right)$ such that $M_{k} \hat{f}=<\hat{f}, \hat{h}>$ for all $f \in l_{p}(\alpha)$. Note that

$$
\left.M_{k}\left(\hat{M}_{z}\right)^{n}\left(z-\lambda_{k}\right)\right)=M_{k}\left(z^{n+1}-\lambda_{k} z^{n}\right)=h_{n+1}-\lambda_{k} h_{n}
$$

for all integers $n \geq 0$. Since $\left.M_{k}\left(\hat{M}_{z}\right)^{n}\left(z-\lambda_{k}\right)\right)=0$, we get $h_{n+1}=\lambda_{k} h_{n}$ and so $h_{n+1}=\lambda_{k}^{n+1} h_{0}^{(k)}$ for all $n \geq 0$. But $\left\{\lambda_{k}^{n}\right\}_{n} \notin l_{q}\left(\alpha^{-1}\right)$ and $h \in l_{q}\left(\alpha^{-1}\right)$, hence $h_{n}=0$ for all $n$ and so $M_{k}=0$. Thus $z-\lambda_{k}$ is cyclic for $k=1, \ldots, m$ and so $\hat{p}(z)$ is a cyclic vector for $\hat{M}_{z}$. The converse case is clear.

Theorem 3. Suppose that $1<p<\infty, w \in \mathcal{M}\left(l_{p}(\alpha)\right), \mathcal{M}\left(\hat{l}_{p}(\alpha)\right)=H^{\infty}$ and $\varphi$ is an analytic self-map of the open unit disc $U$ satisfying $\|\varphi\|_{U}<1$. Also, let $\sum_{n=0}^{\infty} \alpha_{n}^{-q}<\infty$ where $\frac{1}{p}+\frac{1}{q}=1$. If there exists $z_{0} \in U$ satisfying $\hat{w}\left(\varphi_{k}\left(z_{0}\right)\right) \neq 0$ for all $k \geq 0$ and if the set $\left\{\varphi_{k}\left(z_{0}\right): k \geq 0\right\}$ has limit point in $U$, then $e\left(z_{0}\right)$ is a cyclic vector for the operator $\left(M_{w} C_{\varphi}\right)^{*}$ acting on $\hat{l}_{q}\left(\alpha^{-1}\right)$.

Proof. By the property $\sum_{n=0}^{\infty} \alpha_{n}^{-q}<\infty$, each point of $U$ is a bounded point evaluation and the space $\hat{l}_{p}(\alpha)$ consists of functions analytic in the open unit disc $U$. Let the map $L: \mathcal{M}\left(\hat{l}_{p}(\alpha)\right) \rightarrow B\left(\hat{l}_{p}(\alpha)\right)$ be given by $L(\hat{\psi})=\hat{M}_{\hat{\psi}}$. We prove that $L$ is continuous. For this we use the closed graph theorem. Suppose $\hat{\psi}_{n}$ converges to $\hat{\psi}$ in $\mathcal{M}\left(\hat{l}_{p}(\alpha)\right)$ and $L\left(\hat{\psi}_{n}\right)=\hat{M}_{\hat{\psi}_{n}}$ converges to $A$ in $B\left(\hat{l}_{p}(\alpha)\right)$. Then for each $f$ in $l_{p}(\alpha)$,

$$
A \hat{f}=\lim _{n} \hat{M}_{\hat{\psi}_{n}} \hat{f}=\lim _{n} \hat{\psi}_{n} \hat{f} .
$$

Thus $\left\{\hat{\psi_{n}} \hat{f}\right\}_{n}$ is convergent in $\hat{l}_{p}(\alpha)$. Now by the continuity of point evaluations $\hat{\psi}_{n} \hat{f}$ converges pointwise to $\hat{\psi} \hat{f}$ on $U$. So $A \hat{f}$ is analytic and agree with $\hat{\psi} \hat{f}$ on $U$. Hence $A \hat{f}=\hat{\psi} \hat{f}$ and $A=\hat{M}_{\hat{\psi}}$. Therefore $L$ is continuouse and there is a constant $c$ such that $\left\|\hat{M}_{\hat{\psi}}\right\| \leq c\|\hat{\psi}\|_{U}$ for all $\hat{\psi}$ in $\mathcal{M}(\hat{F})$. But $\|\hat{\psi}\| \leq\left\|\hat{M}_{\hat{\psi}}\right\|$ for all $\hat{\psi}$ in $\mathcal{M}(\hat{F})$. Thus $\|\hat{\psi}\| \leq c\|\hat{\psi}\|_{U}$ for all $\psi \in \mathcal{M}\left(l_{p}(\alpha)\right)$. Since $\varphi \in H^{\infty}$ and 
$\mathcal{M}\left(\hat{l}_{p}(\alpha)\right)=H^{\infty}$, we will use $\hat{\varphi}$ instead of $\varphi$. Let $f \in l_{p}(\alpha)$, then $C_{\hat{\phi}} \hat{f}=\hat{f} o \hat{\varphi} \in$ $H^{\infty}$ since $\|\hat{\varphi}\|_{U}<1$. So

$$
\|\hat{f} \circ \hat{\varphi}\| \leq c\|\hat{f} \circ \hat{\varphi}\|_{U} \leq c\|\hat{f}\|_{U},
$$

because $\hat{\varphi}(U) \subseteq U$. On the otherhand, note that for all $f$ in $l_{p}(\alpha),\|\hat{f}\|_{U} \leq \gamma\|\hat{f}\|$ where $\gamma=\sum_{n=0}^{\infty} \alpha_{n}^{-q}$. Now we get $\left\|C_{\hat{\varphi}} \hat{f}\right\| \leq c \gamma\|\hat{f}\|$ which implies that $C_{\hat{\varphi}}$ and so $\hat{M}_{\hat{w}} C_{\hat{\varphi}}$ is bounded. Now, put $A=\hat{M}_{\hat{w}} C_{\hat{\varphi}}$. To complete the proof we show that if for all $k \geq 0,<\hat{g},\left(A^{*}\right)^{k} e\left(z_{0}\right)>=0$, then $\hat{g}$ should be the zero constant function. For this note that

$$
<\hat{g},\left(A^{*}\right)^{k} e\left(z_{0}\right)>=\left(\prod_{i=0}^{k-1} \overline{\hat{w}\left(\hat{\varphi}_{i}\left(z_{0}\right)\right)}\right) \hat{g} \circ \hat{\varphi}_{k}\left(z_{0}\right) .
$$

By the assumptions, clearly we get $\hat{g} \circ \hat{\varphi}_{k}\left(z_{0}\right)=0$ for all $k \geq 0$. Since $\left\{\hat{\varphi}_{k}\left(z_{0}\right)\right.$ : $k \geq 0\}$ has limit point in $U$, it should be $\hat{g}=0$. Thus, $e\left(z_{0}\right)$ is a cyclic vector for the operator $\left(\hat{M}_{\hat{w}} C_{\hat{\varphi}}\right)^{*}$ acting on $\hat{l}_{q}\left(\alpha^{-1}\right)$. This completes the proof.

\section{References}

[1] L. Bagheri and B. Yousefi, Reflexivity of the shift operator on some BK spaces, Rendiconti Del Circolo Matematico Di Palermo, Volume 2013, DOI 10.1007/s12215-013-0143-5 (2013).

[2] M. Mursaleen and A. K. Noman, On some new difference sequence spaces of non-absolute type, Math. Comput. Modelling, 52 (2010), 603-617.

[3] E. Malkowsky, Linear operators in certain BK spaces, Bolyai Society Mathematical Studies, 5 (1996), 259-273.

[4] A. Wilansky, Summability through functional analysis, Mathematics Studies 85, North-Holland, 1984.

[5] B. Yousefi, On the space $\ell^{p}(\beta)$, Rendiconti Del Circolo Matematico Di Palermo, Serie II, Tomo XLIX (2000) 115-120.

[6] B. Yousefi, Unicellularity of the multiplication operators on Banach spaces of formal power series, Studia Math., 147 (2001) 201-209. 